\title{
Use of Mutual Coupling to Decrease Parasitic Inductance of Shunt-Capacitor Filters
}

\author{
J. Bernal, Senior Member, IEEE, M.J. Freire, Senior Member, IEEE, and S.Ramiro
}

\begin{abstract}
In this work we propose and study several new designs of a shunt capacitor filter with two surface-mount technology capacitors. These designs makes use of mutual inductance effects to increase the attenuation provided by the filter in the range of high frequencies where the filter behaves inductively. We provide lumped element circuit models for the proposed designs that allow to identify the key inductive parameters that determine the high-frequency performance of these filters. We obtain the equations relating these parameters to the effective inductance of the filter, which can be used to compare the high frequency behavior of different filter designs. We have fabricated and measured several compact shunt capacitor filters with improved performance at high frequencies. We have found that, compared with a shunt capacitor filter with one capacitor, a proper filter design with two capacitors can easily increase in 15-20dB the high-frequency attenuation provided by the filter. This design also outperforms by $10-15 \mathrm{~dB}$ a traditional shunt capacitor filter with two capacitors closely placed. Moreover, this improvement is obtained with no increase in size, cost or time of design of the filter.
\end{abstract}

Index Terms-EMI filters, shunt-capacitor filters, parasitic inductance, high-frequency effects

\section{INTRODUCTION}

$\mathbf{L}$ OW pass filters are today key components in helping electronic circuits to meet the applicable EMC regulations. In general a filter with high insertion loss can be achieved by using a sufficiently high number of filter components and stages. However, practical and economic considerations make it desirable to keep EMI filters as simple, cheap and compact as possible [1].

Shunt capacitors are widely employed in many applications as a low-cost, compact and effective low-pass filter. However, the high frequency performance of a shunt capacitor filter is limited by the the mutual inductance between the input and output loops of the capacitor, that causes the capacitor to behave inductively above its frequency of resonance [1][3]. Up to a point, this issue can be dealt with by using design rules as minimizing the length and height above the ground plane of the trace that connects the capacitor to the via or using multiple vias [2], [4]. To further improve the performance of the shunt capacitor filter it is necessary to implement methods to cancel the parasitic inductance of the shunt capacitor [5][10]. These works make use of magnetic coupling between the

J. Bernal is with the Department of Física Aplicada III, Universidad de Sevilla, Spain, e-mail: jbmendez@us.es

M.J. Freire is with the Department of Electronics and Electromagnetism, Universidad de Sevilla, Spain, e-mail: freire@us.es

S.Ramiro is with Skylife Engineering, Seville, Spain, email: sebastianraut@skylife-eng.com

This work has been supported by the Spanish Ministerio de Economía y Competitividad (project TEC2014-54097-R).

Manuscript received —-, 2015; revised —— 2015. traces connected to the terminals of one or several capacitors to create negative effective inductances that counteract the parasitic inductance of the capacitors. This magnetic coupling is achieved by means of air-core winding printed on the circuit board or discretely constructed. However, these techniques have been designed for physically large shunt capacitors for power applications. Small filters intended to operate at much higher frequencies (typically well above $100 \mathrm{MHz}$ ) as those often required in modern digital circuits usually make use of surface-mount technology (SMT) shunt capacitors connected between the signal trace and the return plane of a printed circuit board (PCB). In this applications coupled coils are not practical due not only to space restrictions but also to the fact that parasitic capacitance limits the effectiveness of coupled windings at frequencies above a few tens of megahertzs [6].

Reduction of parasitic inductance of shunt SMT capacitors by using capacitors with three and four leads have been reported in [3], [11]. Also, [12] demonstrates that by placing two shunt capacitors in parallel separated an appropriate distance it is possible to attain a significant improvement in the attenuation of the filter. Recently, in [13], a new method has been presented that successfully adapt the canceling inductance scheme in [6] to shunt capacitor filters with SMT capacitors. This design method allows for canceling the parasitic inductance of the shunt capacitors by using magnetic coupling between coplanar traces and it has been more recently generalized to take advantage as well of magnetic coupling between vias in PCBs with more than two layers [14]. Results in [13] show that an effective cancellation of the parasitic inductance of shunt SMT capacitors can be achieved at the cost of increasing the size of the filter. Also, some effort is required to design the coupled traces that provide inductance cancellation. A cost-effective alternative has been recently presented by the authors of this paper in [15]. In that work, the shunt path itself is coupled to a segment of the input or output loops of the filter circuit to provide $8-10 \mathrm{~dB}$ of extra attenuation at high frequencies.

In the present work we show that even better results can be obtained for shunt capacitor filters with two SMT capacitors. We demonstrate that in these filters it is possible to take advantage of both the mutual couplings between the shunt paths and the signal trace and the mutual coupling between the shunt paths themselves, which is a limiting parameter in many cases [12]. We carry out a comprehensive study of the impact of mutual couplings on the performance of these filters and present circuit models that accounts for the improvements obtained with the different filter designs that we propose. Several test boards have been fabricated and measured to verify the accuracy of these circuit models and to determine 


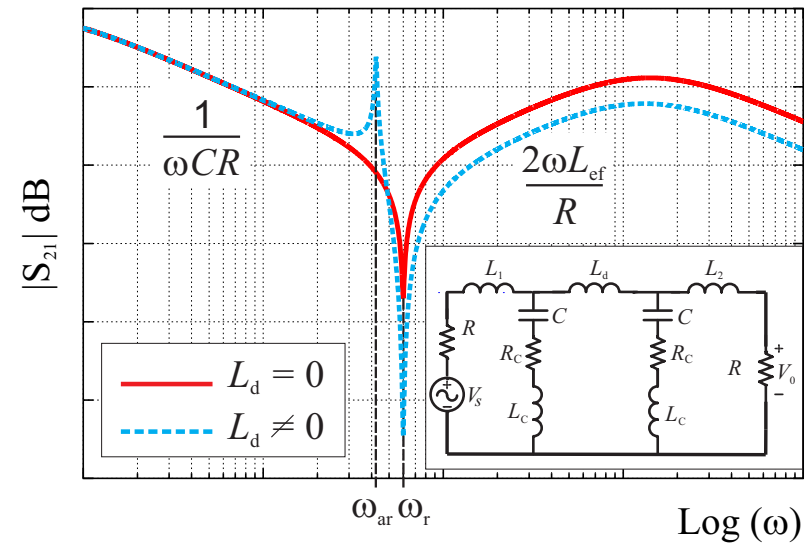

Fig. 1. $\left|S_{21}\right|$ of a simple model of a filter with two capacitors.

\begin{tabular}{|l|c|c|}
\hline Case & $L_{r}=1 / C \omega_{r}^{2}$ & $L_{a r}=1 / C \omega_{a r}^{2}$ \\
\hline \hline One capacitor & $L_{c}$ & - \\
\hline $\begin{array}{l}\text { One capacitor } \\
\text { coupled to trace } \\
{[15]}\end{array}$ & $L_{c}-M_{t}$ & - \\
\hline Filter of Fig.1 & $L_{c}$ & $L_{c}+\frac{L_{d}}{2}$ \\
\hline
\end{tabular}

TABLE I

INDUCTANCES CORRESPONDING TO THE RESONANT AND ANTI-RESONANT FREQUENCIES, $\omega_{r}$ AND $\omega_{a r}$, OF THE FILTER OF FIG.1. ALSO, INDUCTANCE CORRESPONDING TO $\omega_{r}$ OF A FILTER WITH ONE CAPACITOR OF CAPACITANCE $C$

the actual improvement provided by this design strategy. We show that it is possible to obtain a significant increase of the high-frequency attenuation provided by the filter with no increase of its size, cost or time of design.

\section{ANALYSIS}

The physical implementation of a shunt capacitor filter necessarily introduces a mutual inductance between the input and output loops of the circuit [3], [12]. This mutual inductance can be modeled as a series inductance and it is usually referred to as the ESL (equivalent series inductance) of the capacitor. Also, if the traces or leads connecting the capacitor to the input and output ports are electrically short they can be modeled by another two self-inductances that actually accounts for the magnetic flux that does not link the input and output loops. These two self-inductances may be negligible if the ESL of the capacitor is sufficiently high [11]. The presence of the ESL causes the impedance of the capacitor to increase with frequency above the self-resonance frequency, thus degrading the performance of the shunt capacitor filter [2], [3]. It is wellknown that a filter with a better response at high frequency can be obtained by using two or more shunt capacitors in parallel, instead of only one [12]. Alternatively, some improvement in the high frequency response of a single capacitor filter can be obtained by exploiting the magnetic coupling of the capacitor's shunt path to the signal trace [15]. To understand the improvement provided by the filter designs proposed in this work it is illustrative to start by analyzing a simple model of a shunt capacitor filter with two capacitors and compare its features with those of a filter with only a shunt capacitor.

\section{A. Simple model of a filter with two capacitors}

Fig. 1 shows a schematic of a filter that could model in a first approximation a filter with two SMT capacitors connected in parallel to the signal trace and separated a distance $d$. A picture of the physical realization of such a filter is shown in the upper part of Fig.2. In the schematic of Fig.1 an inductance $L_{c}$ and a resistor $R_{c}$ are placed in series with each capacitor to account for the ESL and the equivalent series resistance (ESR) of each capacitor. In that schematic, the filter is excited by a source whose output resistance is matched to the load $R=R_{S}=R_{L}$. The inductance $L_{d}$ in that schematic accounts for the net partial inductance of the portion of line connecting both capacitors, while $L_{1}$ and $L_{2}$ model the electrically short microstrip lines connecting the filter to the source and the load, as explained above. The performance of this filter can be evaluated by calculating the magnitude of its $S_{21}$ scattering parameter. The graph in Fig.1 represents an example of the response of this low-pass filter with two capacitors for two different cases: $L_{d}=0$ (capacitors very close to each other) and $L_{d} \neq 0$. Several aspects stand out regarding Fig.1:

- At low frequencies the attenuation increases with frequency $\left(\left|S_{21}\right| \approx 1 /(\omega C R)\right)$ as expected.

- Due to the ESL of the capacitors the filter has a resonant frequency, $\omega_{r}$.

- Above the resonant frequency there is a wide frequency range were the $S_{21}$ parameter increases proportionally to frequency and to $L_{e f}$, where $L_{e f}$ is the effective inductance of the filter above its resonant frequency. Of course, $L_{e f}$ must be kept as small as possible for achieving good attenuation at high frequencies.

- For $L_{d} \neq 0$ the $\left|S_{21}\right|$ curve presents a local maximum at a given frequency termed anti-resonant frequency, $\omega_{a r}$. This anti-resonance effect does not appear in single capacitor filters and it is related to a series LC resonance corresponding to a current circulating in the central loop created by the two capacitors of the filter and $L_{d}$.

- The filter with $L_{d} \neq 0$ presents a very deep minimum at the resonant frequency $\omega_{r}$. This is due to the existence of a second order zero of $\left|S_{21}\right|$ at $\omega_{r}$ that does not appear in the $L_{d}=0$ case, since in this case $\omega_{a r}=\omega_{r}$, resulting in no anti-resonance and a single order minimum.

- At very high frequencies $\left|S_{21}\right|$ stop increasing and even can start to decrease again. This situation is reached when the impedances of $L_{1}$ and $L_{2}$ become of the same order of magnitude as $R$. In many practical cases this corresponds to frequencies near those at which the line is no longer electrically short [15].

Table I shows $L_{r}$ and $L_{a r}$, namely the inductances that corresponds to the resonant and anti-resonant frequencies of the filter in Fig. 1 and of the filter with only one capacitor that can be coupled or uncoupled to the trace. In the expressions in Table I $M_{t}$ is the mutual inductance between the shunt path and the trace [15]. For a filter with one capacitor the resonant inductance coincides with the effective inductance: $L_{e f}=L_{r}$. 


\begin{tabular}{|l|c|c|}
\hline Case & $L_{r}=1 / C \omega_{r}^{2}$ & $L_{a r}=1 / C \omega_{a r}^{2}$ \\
\hline \hline Filter of Fig.2 & $L_{c} \pm \sqrt{M_{c}\left(M_{c}-L_{d}\right)}$ & $L_{c}+\frac{L_{d}}{2}-M_{c}$ \\
\hline Filter of Fig.3 & $L_{c}+M_{c}-2 M_{t}$ & - \\
\hline Filter of Fig.4 & $L_{c}^{\prime}-M_{c} \pm \sqrt{M_{c}\left(M_{c}+L_{d}^{\prime}\right)}$ & $L_{c}^{\prime}+\frac{L_{d}^{\prime}}{2}$ \\
\hline
\end{tabular}

TABLE II

INDUCTANCE CORRESPONDING TO THE RESONANT AND ANTI-RESONANT FREQUENCIES, $\omega_{r}$ AND $\omega_{a r}$, OF THE FILTERS WITH TWO EQUAL CAPACITORS OF CAPACITANCE $C$ PROPOSED IN THIS WORK. IN THESE EQUATIONS: $L_{d}^{\prime}=L_{d}-2 M_{t}$ AND $L_{c}^{\prime}=L_{c}-M_{t}+M_{c}$.

However, this is not the case for filters with two capacitors. In general, $L_{e f}$ can be calculated by circuit analysis of the lumped element circuit model of the filter by neglecting the impedance of the capacitors (and their ESR, $R_{c}$ ) with respect to $L_{c}$, and by considering that the frequency is still sufficiently low as to allow us to neglect the reactance of the line against the load and source resistances: $\omega L_{i} \ll R$, where $i=1,2$. For $R=50 \Omega$ this condition is met in practice in a wide range of frequencies above the frequency of resonance, as we will see in section III. For the filter in Fig.1 we have:

$$
L_{e f}=\frac{L_{c}}{2\left(1+\frac{L_{d}}{2 L_{c}}\right)}
$$

From (1) it can be seen that for $L_{d}=0$ the effective inductance of the filter with two capacitors is $L_{c} / 2$, i.e. one half of that of the filter with one capacitor. This simply reflects the fact that we have two $L_{c}$ in parallel. However, note that a non-negligible $L_{d}$ further increases the attenuation of the filter, although it also introduces an anti-resonance peak. Measurements reported in section III and in [12] confirm that for low and moderate $d$ values the anti-resonance peak is damped by the ESR of the capacitors. However, note that when $L_{d}$ increases the characteristic impedance of the resonant LC circuit (namely, $Z_{c}=\sqrt{2\left(L_{d}+2 L_{c}\right) / C}$ ) increases and therefore the damping factor of the RLC resonant circuit decreases. This results in an increase of the magnitude of the anti-resonant peak of $\left|S_{21}\right|$ as $d$ increases. This may be an issue if the anti-resonant frequency happens to coincide with a harmonic intended to be filtered out.

Summing up, placing two capacitors in parallel effectively decreases the effective inductance of the filter at high frequencies when comparing with a filter with only one capacitor. This effect is due not only to the fact that the parasitic inductances of the two capacitors are in parallel but also to the inductance $L_{d}$ between the two capacitors. However, increasing the distance between the capacitors also introduces an anti-resonant peak in $\left|S_{21}\right|$.

\section{B. Filters with two capacitors and mutual coupling}

The simple circuit model of Fig.1 fails in that it ignores the effects of magnetic coupling between current paths. To refine the model of the filter with two capacitors, let's focus on Fig.2. Because the capacitor's paths in this filter are parallel to each other, it is possible for some of the magnetic field lines surrounding one of the capacitors to encircle also the
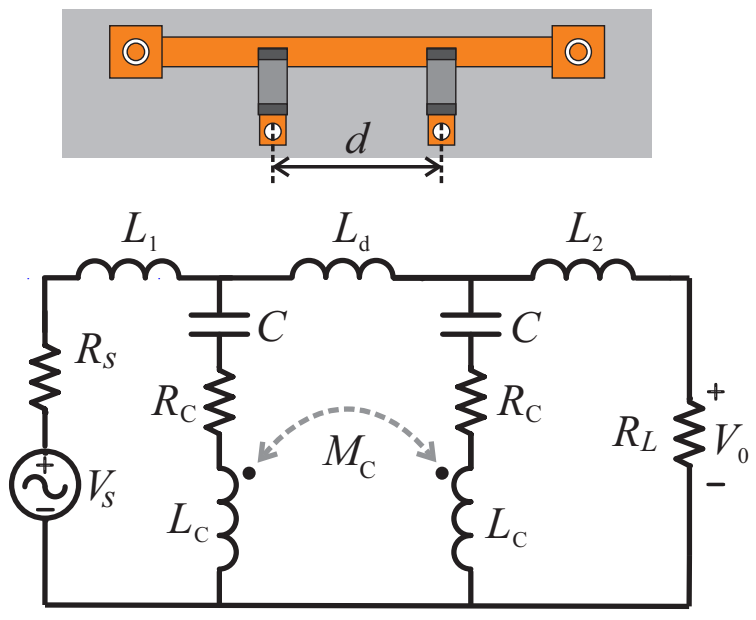

Fig. 2. A filter consisting of two SMT capacitors separated a distance $d$ placed perpendicularlly to the trace (top view) along with its corresponding lumped element circuit schematic.

\begin{tabular}{|l|l|}
\hline Case & $L_{e f}$ \\
\hline \hline Filter of Fig.1 & $\frac{L_{c}}{2\left(1+\frac{L_{d}}{2 L_{c}}\right)}$ \\
\hline Filter of Fig.2 & $\frac{L_{c}+M_{c}\left(\frac{L_{d}}{L_{c}-M_{c}}+1\right)}{2\left(1+\frac{L_{d}}{2\left(L_{c}-M_{c}\right)}\right)}$ \\
\hline Filter of Fig.3 & $\frac{1}{2}\left(L_{c}+M_{c}-2 M_{t}\right)$ \\
\hline Filter of Fig.4 & $\frac{L_{c}^{\prime}-M_{c}\left(\frac{L_{d}^{\prime}}{L_{c}^{\prime}}+2\right)}{2\left(1+\frac{L_{d}^{\prime}}{2 L_{c}^{\prime}}\right)}$ \\
\hline
\end{tabular}

TABLE III

EFFECTIVE INDUCTANCES OF THE SHUNT CAPACITOR FILTERS WITH TWO EQUAL CAPACITORS OF CAPACITANCE $C$ ANALYZED AND PROPOSED IN THIS WORK. IN THESE EQUATIONS: $L_{d}^{\prime}=L_{d}-2 M_{t}$ AND

$$
L_{c}^{\prime}=L_{c}-M_{t}+M_{c}
$$

other capacitor, thus giving rise to a mutual coupling between the shunt paths denoted in the schematics of Fig.2 by $M_{c}$. Since $M_{c}$ accounts for the inductive coupling of the noise current flowing through the first capacitor to the output loop of the filter, the presence of $M_{c}$ is expected to undermine the performance of the filter [12]. This can also be looked at by considering that the two inductances $L_{c}$ are now coupled to form a common-mode choke, which opposes the current flowing simultaneously through both shunt paths to the return plane. The negative impact of $M_{c}$ in the high frequency response of this filter can be confirmed by comparing the equations for $L_{e f}$ that can be obtained by analyzing the schematics of Fig.1 and Fig.2. To facilitate the comparison, these two equations are gathered in Table III along with the equations for the $L_{e f}$ of the alternative filter designs that we are going to analyze in this section. An inspection of the formula for $L_{e f}$ of the filter of Fig.2 shows that the performance of this filter can be optimized by increasing as much as possible the distance between capacitors $d$, since this causes $L_{d}$ to increase and $M_{c}$ to decrease, thus decreasing $L_{e f}$. However, 


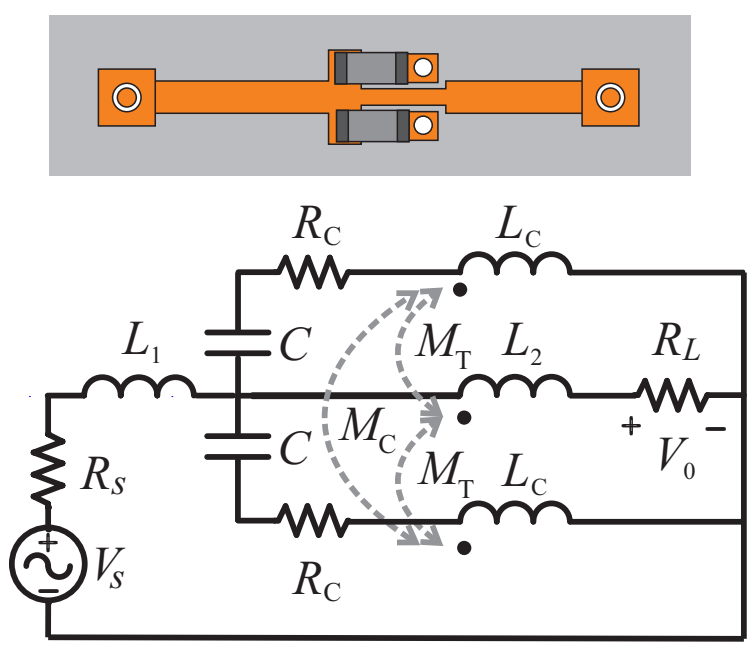

Fig. 3. A filter consisting of two SMT capacitors placed parallel to the trace (top view) along with its corresponding lumped element circuit schematic.

increasing $d$ affects negatively the filter compactness, which can not be always afforded in overcrowded PCBs. Moreover, as mentioned before, the increase of $d$ introduces an antiresonance peak whose magnitude increases with the increasing $L_{d}$. The anti-resonant frequency for the the filter of Fig.2 can be obtained from the $L_{a r}$ inductance, which is included in Table II along with those for the alternative filter designs that are going to be studied in this section. Table II also shows $L_{r}$ for all these filters. From equations in Table II it can be seen that for the filter of Fig.2 the presence of a non-negligible $M_{c}$ causes the double zero of $\left|S_{21}\right|$ at $\omega_{r}$ to split in two single zeros yielding two frequencies of resonance. Moreover, if $L_{d}>M_{c}$ the frequency of resonance become a pair of complex and complex conjugate values if the capacitors are sufficiently far away from each other. However, in practice, when the capacitors are taken apart, $M_{c}$ becomes zero very quickly and this effect is only noticeable as a very deep minimum of the $\left|S_{1} 2\right|$ curve at $\omega_{r}$ caused by two very close zeros. The presence of a deep minimum and of an anti-resonant peak results in a $\left|S_{21}\right|$ curve for the filter of Fig.2 which is very sensitive to the modification of any of the parameters of the filter. In section III we will take advantage of this fact to extract the inductive parameters of the structure $\left(L_{d}, L_{c}\right.$ and $\left.M_{c}\right)$ by means of curve fitting. This will allow us to estimate these parameters and to understand the role played by them in the performance of the alternative filter designs that we present on the remainder of this section.

The first alternative design for a two-capacitor's filter is shown in Fig.3. This design is based upon the idea reported in [15] for a shunt capacitor filter with only one capacitor. Basically, it consist in taking advantage of the mutual inductance created between the shunt path and the signal trace. Table I shows that for the filter with one capacitor the effective inductance decreases when a mutual inductance $M_{t}$ appears between the shunt path and the trace (for this filter $L_{e f}=L_{r}$, as already mentioned). In [15] it is demonstrated that for SMT capacitors this can lead to an increase of $5-10 \mathrm{~dB}$ in the attenuation of the filter at frequencies above the resonant
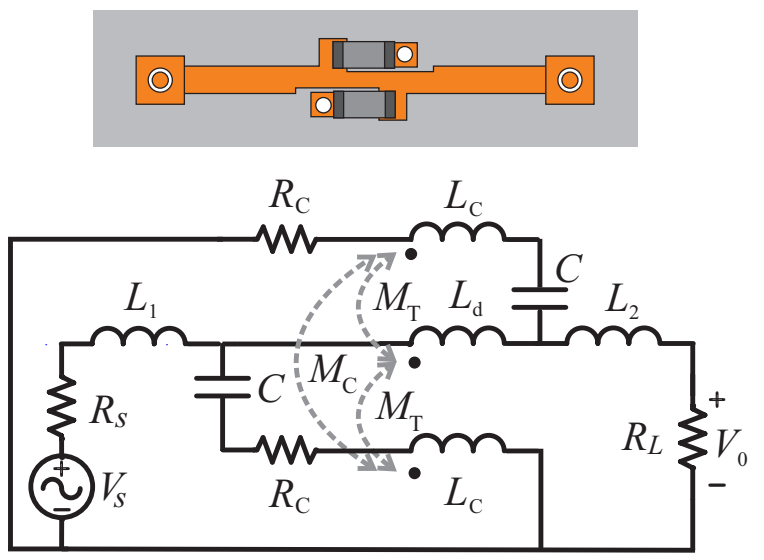

Fig. 4. Top view of a filter with two SMT capacitors placed parallel to the trace and in opposite directions along with its corresponding lumped element circuit schematic.

frequency. The idea here is to increase the effective shuntpath-to-trace coupling by splitting the shunt path into two shunt paths parallel to the trace. This is denoted by the two mutual inductances $M_{t}$ appearing in the schematic in Fig.3. In principle, using a thin trace as close to the capacitors as possible will help to increase $M_{t}$ and, therefore, to improve the attenuation provided by the filter. However, note that placing the two capacitors very close to each other will make it possible for some of the magnetic field lines encircling one of the capacitors to encircle also the other one. This will introduce a mutual coupling between the shunt paths, which is accounted for by the mutual inductance $M_{c}$ in Fig.3. This coupling will degrade the performance of the filter due to the same common-mode choke effect that affects the filter of Fig.2. This is confirmed by the equation of the effective inductance of the filter of Fig.3, shown in Table III. Note that in that equation $L_{c}$ appears multiplied by a $1 / 2$ factor, which is related to the use of two equal inductances in parallel, but this factor does not affect the mutual inductance $M_{t}$ which acts decreasing $L_{e f}$ with a greater relative impact that for a single capacitor filter. However, the coupling between the shunt paths, accounted for by $M_{c}$, increases $L_{e f}$, thus degrading the high-frequency performance of the filter. The actual impact of $M_{c}$ in the performance of this filter will be investigated by fabricating, measuring and comparing several filter prototypes in section III. In spite of the possible negative effect of $M_{c}$, an advantage of this filter design is that because there is no distance between the capacitors, $L_{d}=0$ and the $\left|S_{21}\right|$ curve of the filter will present no anti-resonance peak.

To overcome the negative effect of the mutual coupling between shunt paths that appears in the filter of Fig. 2 we present an alternative filter configuration in Fig.4. In that design the two shunt paths are also coupled to the signal trace, but they are connected in such a way that the high frequency currents diverted from the trace to the return plane through those shunt paths flow in opposite directions in one capacitor with respect to the other. This creates a mutual coupling between these two current paths that decreases the net impedance seen by the currents flowing simultaneously through the shunt paths. In other words, comparing with Fig.2 and Fig.3, the design 


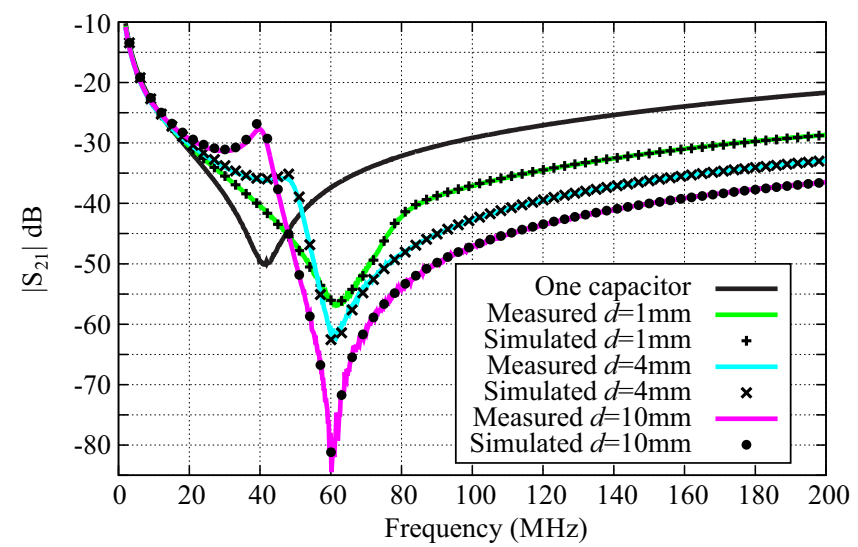

Fig. 5. Measured and simulated results for the filter with two capacitors of Fig. 2 for different distances between the capacitors compared with measures for a single $10-\mathrm{nF}$ shunt capacitor.

\begin{tabular}{|c|c|c|c|c||c|}
\hline Case & $L_{c}$ & $L_{d}$ & $M_{c}$ & $L_{e f}$ & $\Delta\left|S_{21}\right|(\mathrm{dB})$ \\
\hline$d=1 \mathrm{~mm}$ & 1.22 & 0.46 & 0.54 & $\mathbf{0 . 7 9}$ & 4.6 \\
\hline$d=4 \mathrm{~mm}$ & 1.45 & 1.64 & 0.08 & $\mathbf{0 . 5 1}$ & 9.1 \\
\hline$d=10 \mathrm{~mm}$ & 1.47 & 3.58 & 0.02 & $\mathbf{0 . 3 4}$ & 12.8 \\
\hline
\end{tabular}

TABLE IV

INDUCTIVE PARAMETERS (IN NH) OF THE CIRCUIT MODEL OF FIG.2 OBTAINED BY CURVE FITTING OF THE RESULTS OF FIG.5. LAST COLUMN: IMPROVEMENT OF $\left|S_{21}\right|$ MEASURED AT 200MHZ WITH RESPECT TO THE FILTER WITH ONE 10-NF CAPACITOR.

of Fig.4 inverts the dots of the transformer that models the coupling between the two inductances $L_{c}$. In this way the common-mode choke effect appearing in the filters of Fig.2 and Fig. 3 is replaced by an opposite effect which favors the current flowing through both capacitors towards the return plane. Also, the design of Fig.4 introduces a small inductance, $L_{d}$, between the two points of connection of the capacitors to the signal trace. As we have already seen, this inductance improves the high-frequency attenuation of the filter at the cost of introducing an anti-resonant peak on the $\left|S_{21}\right|$ curve. However, because in this case $L_{d}$ is kept necessarily small it will not cause a noticeable anti-resonance effect, as we will show in section III.

The potential benefits of this filter are confirmed by circuit analysis. A close inspection of the equation of the effective inductance of the filter of Fig.4, shown in Table III, reveals that in this case the effect of $M_{c}$ is to decrease, in instead of increase, the effective inductance of the filter, thus contributing to increase the attenuation provided by the filter at high frequencies. Also $M_{t}$ and $L_{d}$ increase the filter attenuation. Therefore, all the self- and mutual inductances introduced in this design are expected to have a positive impact on the performance of the filter. Note also in Table II that $M_{c}$ causes the resonant frequency of this filter to split into two real values. As we will see in section III, for practical values of $L_{d}, M_{c}$ and $M_{t}$ this will result on an increase of the apparent frequency of resonance, which is actually the joint effect of two very close resonances and, consequently, it will appear as a flat minimum of the $\left|S_{21}\right|$ curve.

\section{RESULTS}

In this section we present measured results of several test boards that has been designed and fabricated to verify the improvement actually provided by the alternative filter designs proposed in section II. This will also allow us to check the accuracy of the circuit models of the filters presented in that section. All the filtering test boards described in this section have been mounted on a 1.6-mm thick FR4 substrate. Each low-pass filter is made up of two $4.7 \mathrm{nF}$ capacitor connecting a $30-\mathrm{mm}$-long and $1.5-\mathrm{mm}$-width microstrip trace to a signal return plane. Two SMA connectors at both ends of each microstrip trace allow for the magnitude of the $S_{21}$ scattering parameter (the insertion gain of the filter) to be measured with a network analyzer. We have used an Agilent Fieldfox N9912A.

The first filter that we analyze in this section is that of Fig.2. We have fabricated three prototypes of that filter that only differ in the distance between the two capacitors $(d=1 \mathrm{~mm}$, $4 \mathrm{~mm}$ and $10 \mathrm{~mm})$. Fig.5 shows the measured $\left|S_{21}\right|$ for these filters along with that of a filter with only one $10-\mathrm{nF}$ capacitor (approximately same net capacitance). Note that the filter with two widely separate capacitors outperforms by $15 \mathrm{~dB}$ the filter with only one capacitor. This agrees with results reported in [12]. Results in Fig.5 not only make it possible a direct comparison of the performance of that filter with that of the alternative designs proposed in this work, but also will allow us to estimate typical values of the inductive parameters $\left(M_{c}, L_{c}\right.$ and $\left.L_{d}\right)$ that account for the high-frequency behavior of this filter and of the alternative filters proposed in this work. This will also allow us to calculate the exact (negative) impact of $M_{c}$ in the high-frequency behavior of this filter. This calculation will help to understand and evaluate the effect of $M_{c}$ on the alternative filters proposed in this work.

As explained in section II, the exact shape of the $\left|S_{21}\right|$ curve is very sensitive to small changes of $M_{c}, L_{c}$ and $L_{d}$. This makes easy to determine the value of these parameters by curve fitting. In Fig.5 we have represented, along with the measured results, simulated results for $\left|S_{21}\right|$ obtained by using the lumped element circuit model of Fig.2 and a curve-fitting algorithm [16]. The values obtained for $M_{c}$, $L_{c}$ and $L_{d}$ are given in Table IV, while the ESR of the capacitors has been found to be $R_{c} \approx 0.09 \Omega$ in all cases. In Table IV we also include the effective inductance of each filter calculated by using (1). Note that, by comparison, the effective inductance of the filter with only one $10 \mathrm{nF}$-capacitor is: $L_{e f}=L_{c}=1.51 \mathrm{nH}^{1}$. Therefore, a reduction of a $77 \%$ in $L_{e f}$ is achieved by the filter with $d=10 \mathrm{~mm}$. From results in Table IV it can also be seen that the increasing $L_{d}$ values are quite consistent with the $0.41 \mathrm{nH} / \mathrm{mm}$ value of the p.u.l. inductance of the microstip trace above the ground plane. These results also demonstrate that a significant mutual coupling (up to $\sim 0.5 \mathrm{nH}$ ) can appear between the shunt paths of two SMT capacitors when closely placed. In fact, it is easy to evaluate the impact of $M_{c}$ on the performance of the filter

\footnotetext{
${ }^{1}$ This have been obtained also by curve fitting with simulated results (not shown) using the lumped element circuit model of the filter with only one capacitor and no mutual couplings with $C=10 \mathrm{nF}$ and $R_{c}=0.08 \Omega$.
} 
a)

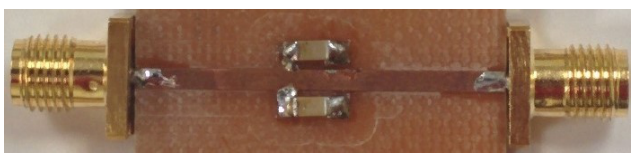

b)

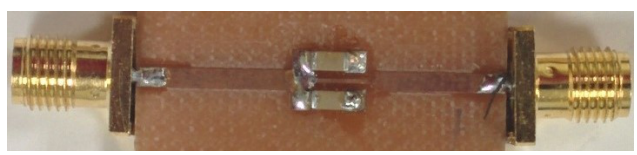

Fig. 6. Two designs of a filter with two 4.7-nF capacitors corresponding to the scheme of Fig.3. Case a): trace of width $1.8 \mathrm{~mm}$ between capacitors. Case b): trace of width $0.6 \mathrm{~mm}$ between capacitors.

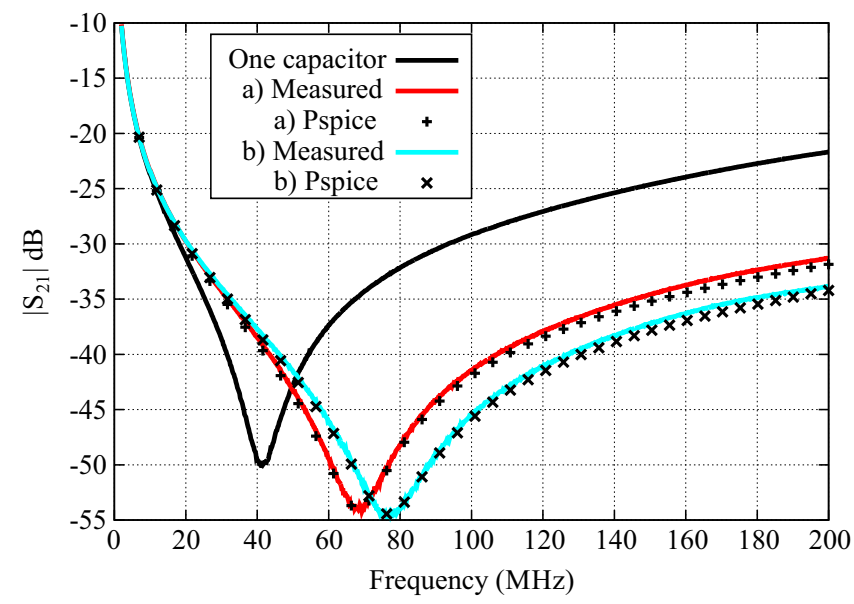

Fig. 7. Measured results for the filters of Fig.6 compared with those for a single $10-\mathrm{nF}$ capacitor filter.

\begin{tabular}{|c|c|c|c|c||c|}
\hline Case & $L_{c}$ & $M_{t}$ & $M_{c}$ & $L_{e f}$ & $\Delta\left|S_{21}\right|(\mathrm{dB})$ \\
\hline a) & 1.6 & 0.27 & 0.1 & $\mathbf{0 . 5 8}$ & 9.6 \\
\hline b) & 1.6 & 0.49 & 0.3 & $\mathbf{0 . 4 6}$ & 12.1 \\
\hline
\end{tabular}

TABLE V

INDUCTIVE PARAMETERS (IN NH) OF THE CIRCUIT MODEL OF FIG.3 OBTAINED BY CURVE FITTING OF THE RESULTS OF FIG.7. LAST COLUMN: IMPROVEMENT OF THE $\left|S_{21}\right|$ MEASURED AT $200 \mathrm{MHZ}$ WITH RESPECT TO THE FILTER WITH ONE 10 -NF CAPACITOR.

simply by taking $M_{c}=0$ in the circuit simulator (Pspice) while leaving unaltered the rest of the parameters of the circuit model of the filter. By comparing these simulated results with the measurements of Fig.5 we have found that for $d=1 \mathrm{~mm}$ the effect of $M_{c}$ is to decrease the attenuation provided by the filter at high frequencies by almost $5 \mathrm{~dB}$, while it increases less than $2 \mathrm{~dB}$ this attenuation when $d=4 \mathrm{~mm}$ and only $0.6 \mathrm{~dB}$ for $d=10 \mathrm{~mm}$. Therefore, a key point to enhance the high frequency response of a compact shunt capacitor filter with two capacitors is to reduce or eliminate the negative effect of the magnetic coupling between shunt paths.

The first alternative proposed here is to design a filter where the effect of $M_{c}$ is counteracted by the magnetic coupling of the shunt paths to the trace, as shown in Fig.3. To check the performance of this design we have fabricated two filters with two 4.7-nF SMT capacitors, shown in Fig.6. In case a) the trace between the capacitors keeps its with of $1.8 \mathrm{~mm}$. In case b) the trace width between the capacitors is thinner $(0.6 \mathrm{~mm})$ so as to increase the coupling between the capacitors and the trace. In Fig.7 measured results for $\left|S_{21}\right|$ of these two filters are compared with those of a conventional filter consisting in a single $10-\mathrm{nF}$ capacitor perpendicular to the trace. The improvement obtained in the high-frequency insertion gain is included in Table V. These results show that the filter with the two capacitors coupled to the trace outperforms the single capacitor filter at high frequencies by almost $10 \mathrm{~dB}$ for a width trace and by $12 \mathrm{~dB}$ for the thin trace case. When comparing these results with results in Fig.5 for the filter of Fig.2 it can be observed that coupling the capacitors to the trace provides an extra 3-5dB of attenuation at high frequencies with respect to the case in Fig. 5 where $d=1 \mathrm{~mm}$. Nevertheless, if the capacitors are widely separated $(d=10 \mathrm{~mm})$ the filter of Fig. 2 outperforms that of Fig. 3 by the more or less the same amount of 3-5dB. However note that separating the capacitors sacrifices the compactness of the filter, which is maintained in the design of Fig.3. An additional advantage of this latter design is that it presents no anti-resonance peak.

In Fig.7 we have included simulated results obtained with Pspice using the lumped elements circuit model of Fig. 3 and the inductive parameters of Table $\mathrm{V}$, obtained by curve fitting (we have also taken $L_{1}=L_{2}=6.15 \mathrm{nH}$, although the effect of $L_{1}$ and $L_{2}$ is negligible in that range of frequencies). An objective comparison of measured and simulated results can be carried out by using the Feature Selective Validation (FSV) method [17], [18]. By using FSV we have obtained GDM=0.12 ("Very Good" agreement) with GRADE=SPREAD=3 for the two pairs of data set represented in Fig.7. The good agreement achieved between measured and simulated results demonstrates that the lumped element circuit model of Fig.3 is able to account for the increase of the resonance frequency and of the high-frequency filter attenuation due to the coupling of the capacitors to the trace. In particular, it shows that decreasing the distance between the two capacitors increases both $M_{t}$ and $M_{c}$ more or less in the same amount. This improves the filter performance in accordance with the equation for $L_{e f}$ of this filter shown in Table III. Regarding the inductive parameters of Table $\mathrm{V}$, it should be pointed out that, as opposite to the case of the previous filter, for this filter the $\left|S_{21}\right|$ curves in Fig.7 are quite simple and therefore they can be approximated with more than one set of parameters. This makes it necessary to use parameters consistent with those of Table IV as departing point in the curve-fitting algorithm. Nevertheless, our aim in obtaining these parameters is to demonstrate that a simple circuit model as that in Fig. 3 can account for the high-frequency behavior of this filter and to provide an approximation to the self and mutual inductances that can be expected in a filter with SMT capacitors of these characteristics. As a final remark, note that the reduction of the trace width in case b) should have an impact in the inductance $L_{2}$ of Fig.3. However, since $L_{e f}$ is independent of $L_{2}$ (see Table III), this small change can be ignored in the circuit model up to several gigahertzs.

Although the design in Fig.3 outperforms that of Fig.2 for close capacitors, a very similar improvement can be obtained if the capacitors are placed perpendicular to the trace but in opposite sides, to avoid the $M_{c}$ coupling. We have checked that by fabricating the filter shown in Fig.8. We have found that this filter provides a high-frequency attenuation (not shown) 


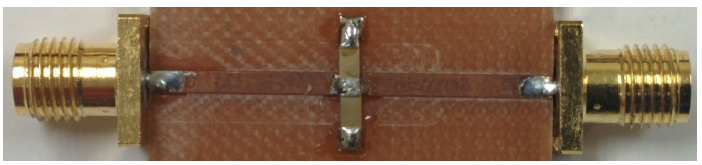

Fig. 8. A filter with two 4.7-nF capacitors at opposite sides of the trace.

\begin{tabular}{|c|c|c|c|c|c||c|}
\hline Case & $L_{d}$ & $L_{c}$ & $M_{t}$ & $M_{c}$ & $L_{e f}$ & $\Delta\left|S_{21}\right|(\mathrm{dB})$ \\
\hline c) & 0.8 & 1.8 & 0.40 & 0.41 & $\mathbf{0 . 5 0}$ & 11.4 \\
\hline d) & 1.65 & 1.8 & 0.45 & 0.47 & $\mathbf{0 . 2 8}$ & 16.7 \\
\hline e) & 0.95 & 1.41 & 0.47 & 0.43 & $\mathbf{0 . 2 5}$ & 18.1 \\
\hline f) & 1.7 & 1.88 & 0.59 & 0.64 & $\mathbf{0 . 2 1}$ & 20.6 \\
\hline
\end{tabular}

TABLE VI

INDUCTIVE PARAMETERS (IN NH) OF THE CIRCUIT MODEL OF FIG.4 OBTAINED BY CURVE FITTING OF THE RESULTS OF FIG.10. LAST COLUMN: IMPROVEMENT OF $\left|S_{21}\right|$ MEASURED AT $200 \mathrm{MHZ}$ WITH RESPECT TO THE FILTER WITH ONE 10-NF CAPACITOR.

which is very close to that of case a) in Fig.7. To understand this, note that because the capacitors are placed perpendicular in instead of parallel to the trace the design of Fig.8 allows for a lower trace-to-via distance for the currents (and hence less $L_{c}$ ) while $M_{t}$ and $M_{c}$ are negligible. In conclusion, results for the filter of Fig.3 demonstrates that this compact design provides some improvement of the high-frequency attenuation of the filter comparing with a traditional shunt capacitor filter with one or two closely placed capacitors. However, mutual coupling between shunt paths limits the improvement that can be obtained in practice.

In Fig. 4 we have presented another alternative filter layout where the capacitors are placed in such a way that the magnetic coupling between the shunt paths improves, in instead of degrades, the performance of the filter. Fig.9 shows four different test boards based upon the filter of Fig.4. Cases c) and d) in Fig.9) are two versions of the design of Fig.4. The section of trace between both capacitors is made thinner in case d) to increase $M_{t}$ [15]. This also increases $M_{c}$ because a thinner trace allows for placing the capacitors closer to each other. In case e) the signal trace makes a zigzag or meander under the capacitors. This is a very simple and effective form of simultaneously increasing $M_{t}$ (because the current on the trace flows under in instead of by the side of the capacitors) and $M_{c}$ (because there is no signal trace between the capacitors and consequently they can be placed closer to each other). Case f) in Fig.9 splits the trace into two thin branches under the capacitors. This will increase $M_{t}$ while allowing also to place the capacitors very close to each other. The improvement of $\left|S_{21}\right|$ at high frequency obtained with these filters with respect to a filter with one 10-nF capacitor are shown in Table VI. Comparing with Table IV it can be seen that the insertion gain of filter d) at high frequencies is almost $4 \mathrm{~dB}$ lower than that provided by the filter of Fig.2 with widely separated capacitors. Note that this is achieved without sacrificing filter compactness and with no significant anti-resonance effect ${ }^{2}$. Fig. 10 and results in Table VI also show that these good results can be further improved with a more careful design of the filter.

\footnotetext{
${ }^{2}$ Note the zigzag of the $\left|S_{21}\right|$ curve of case d) at approximately $50 \mathrm{MHz}$ This is a damped anti-resonance effect that becomes slightly noticeable in this case compared with the others in the same graph due to the relatively high inductance of the thin trace between the capacitors, $L_{d}$.
}

c)

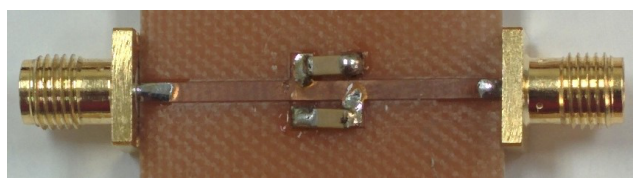

d)

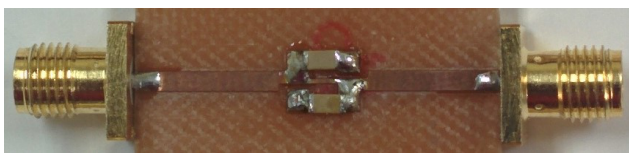

e)

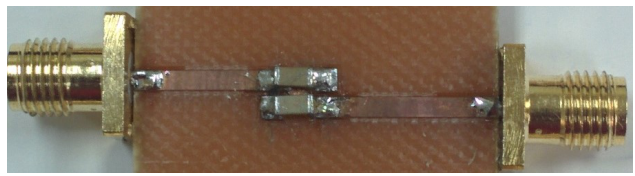

f)

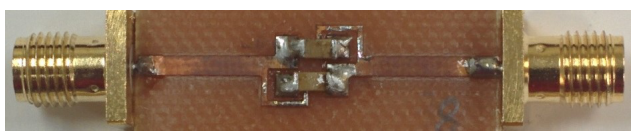

Fig. 9. Four designs of a filter with two 4.7-nF capacitors corresponding to the scheme of Fig.4. Case c): trace of width $1.8 \mathrm{~mm}$ between capacitors. Case d): trace of width $0.6 \mathrm{~mm}$ between capacitors. Case e): trace of width $1.8 \mathrm{~mm}$ meandering under the capacitors. Case f): trace split into two thin $(0.6 \mathrm{~mm})$ traces passing under the capacitors.

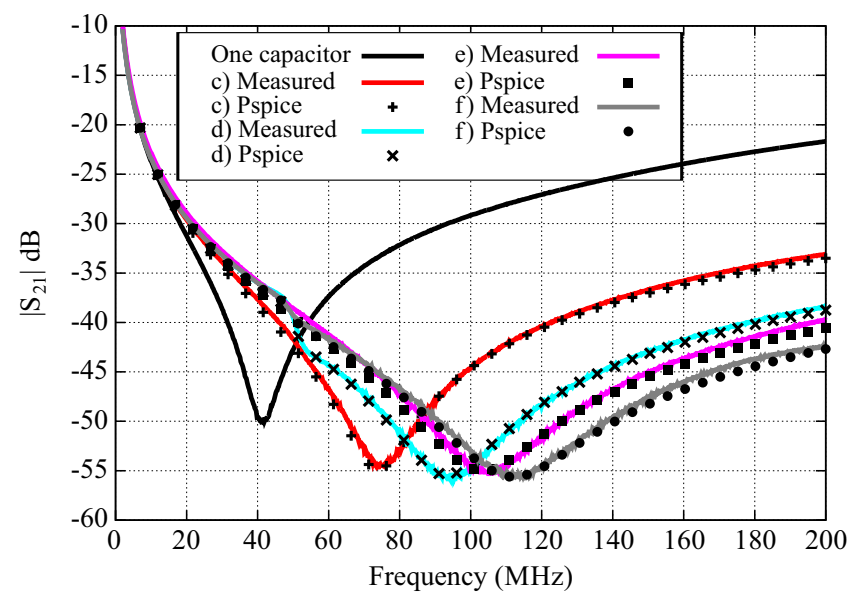

Fig. 10. Measured results for a single 10-nF capacitor filter compared with measured results for the different designs of Fig.9.

In fact, cases e) and f) of Fig.9 provide improvements in the high frequency attenuation of the filter near and above $20 \mathrm{~dB}$. In our opinion, case e) stands out for its simplicity and because it does not require to alter the trace width. That means a very low impact on the characteristic impedance and the resistance of the signal trace.

Simulated results are also represented in Fig.10. These simulated results have been calculated by using the circuit model of Fig. 4 and the inductive parameters shown in Table VI, which have been obtained by curve fitting. We have compared simulated and measured results by using the FSV method [17], obtaining GDM=0.12 with GRADE $=$ SPREAD $=3$ for the four pairs of data set represented in Fig.10. This very good agreement between measured and simulated results suggests that the lumped element circuit of Fig.4 correctly accounts for the physical phenomena that determines the high frequency behavior of this filter. Table VI also gathers the calculated $L_{e f}$ parameters of each version of this filter. It can 
be seen that the minimum value of $L_{e f}$ corresponds to case f). This effective inductance is between seven and eight times lower than the $1.51 \mathrm{nH}$ value of the single capacitor filter.

\section{CONCLUSION}

In this work we have proposed, fabricated and tested several new compact designs of a shunt capacitor filter with two SMT capacitors. Those new designs benefit from magnetic coupling effects to reduce the degradation of the filter response at high frequencies that appears due to the parasitic inductances of the shunt paths. Moreover, these new filter layouts do not require to increase the cost, size or time of design of the filter. We have provided a comprehensive explanation of the influence of mutual inductance in different filter designs with two capacitors. We have presented a lumped element circuit model that accounts for the response of the proposed filters. By comparing the measured results with the circuit models of the filters we have provided approximate values of the mutual inductances that can be achieved with typical SMT capacitors.

We have shown that in conventional filters with two capacitors, the mutual coupling between shunt paths usually degrades filter performance. As a result, the distance between capacitors must be increased to improve the performance of the filter at high frequency, thus sacrificing compactness and introducing an anti-resonant effect that must be controlled. We have proposed an alternative to overcome these drawbacks and to increase filter performance. In particular, we have found and optimum design for a filter with two SMT capacitors where the capacitors are oriented parallel to the trace and in close proximity between them, so that they are magnetically coupled both to the trace and between them, and with shunt currents flowing in opposite directions. This design provides from 8 to $16 \mathrm{~dB}$ of extra attenuation at high frequencies when comparing with a conventional filter configuration with two capacitors, and an improvement of up to $20 \mathrm{~dB}$ comparing with a filter with one capacitor.

\section{REFERENCES}

[1] C. R. Paul, Introduction to electromagnetic compatibility. John Wiley and Sons, 2006.

[2] G. Tang, "Surface mount capacitor loop inductance calculation and minimization," in IEEE International Symposium on Electromagnetic Compatibility, 1998, pp. 505-510.

[3] C. N. Olsen, T. P. Van Doren, T. H. Hubing, J. L. Drewniak, and R. E. DuBroff, "Improving the high-frequency attenuation of shunt capacitor, low-pass filters," in IEEE International Symposium on Electromagnetic Compatibility, 2001, pp. 487-489.

[4] E. Bogatin, Signal and Power Integrity - Simplified, P. Hall, Ed., 2004

[5] T. C. Neugebauer, J. W. Phinney, and D. J. Perreault, "Filters and components with inductance cancellation," IEEE Trans. Ind. Appl., vol. 40, no. 2, pp. 483-491, 2004.

[6] T. Neugebauer and D. Perreault, "Filters With Inductance Cancellation Using Printed Circuit Board Transformers," IEEE Trans. Power Electron., vol. 19, no. 3, pp. 591-602, May 2004.

[7] S. Wang, F. Lee, and W. Odendaal, "Cancellation of Capacitor Parasitic Parameters for Noise Reduction Application," IEEE Trans. Power Electron., vol. 21, no. 4, pp. 1125-1132, Jul. 2006.

[8] B. J. Pierquet, T. C. Neugebauer, and D. J. Perreault, "Inductance compensation of multiple capacitors with application to common- and differential-mode filters," IEEE Trans. Power Electron., vol. 21, no. 6, pp. 1815-1824, 2006

[9] B. Pierquet, T. Neugebauer, and D. Perreault, "A Fabrication Method for Integrated Filter Elements With Inductance Cancellation," IEEE Trans. Power Electron., vol. 24, no. 3, pp. 838-848, Mar. 2009.
[10] H. F. Chen, C. Y. Yeh, and K. H. Lin, "A method of using two equivalent negative inductances to reduce parasitic inductances of a three-capacitor EMI filter," IEEE Trans. Power Electron., vol. 24, no. 12, pp. $2867-$ $2872,2009$.

[11] T. Zeeff, a. Ritter, T. Hubing, and T. VanDoren, "Analysis of a LowPass Filter Employing a 4-Pin Capacitor," IEEE Trans. Electromagn. Compat., vol. 47, no. 1, pp. 202-205, Feb. 2005.

[12] T. Zeeff, T. Hubing, T. Van Doren, and D. Pommerenke, "Analysis of simple two-capacitor low-pass filters," IEEE Trans. Electromagn. Compat., vol. 45, no. 4, pp. 595-601, Nov. 2003.

[13] A. J. McDowell and T. H. Hubing, "Parasitic Inductance Cancellation for Surface Mount Shunt Capacitor Filters," IEEE Trans. Electromagn. Compat., vol. 56, no. 1, pp. 74-82, Feb. 2014.

[14] — "A Compact Implementation of Parasitic Inductance Cancellation for Shunt Capacitor Filters on Multilayer PCBs," IEEE Trans. Electromagn. Compat., vol. 57, no. 2, pp. 257-263, 2015.

[15] J. Bernal, M. Freire, and S. Ramiro, "Simple and Cost-Effective Method for Improving the High Frequency Performance of Surface-Mount Shunt Capacitors Filters," in IEEE International Symposium on Electromagnetic Compatibility, 2015, pp. 372-377.

[16] D. Marquardt, "An algorithm for least-squares estimation of nonlinear parameters," Journal of the Society for Industrial and Applied Mathematics, vol. 11, no. 2, pp. 431-441, 1963.

[17] A. Duffy, A. Martin, A. Orlandi, G. Antonini, T. Benson, and M. Woolfson, "Feature Selective Validation (FSV) for Validation of Computational Electromagnetics (CEM). Part IThe FSV Method," IEEE Trans. Electromagn. Compat., vol. 48, no. 3, pp. 449-459, Aug. 2006.

[18] IEEE Standard for Validation of Computational Electromagnetics Computer Modeling and Simulations, IEEE STD 1597.1, 2008.

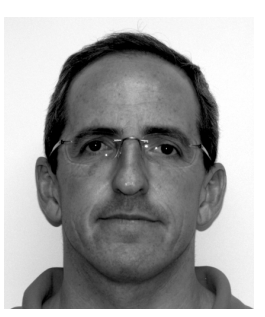

Joaquín Bernal (M'06-SM'14) received the Licenciado and Ph.D. degrees from the University of Seville, Spain, in 1994 and 2000, respectively, both in physics. In 1995, he joined the Department of Electronics and Electromagnetism, University of Seville. In 1998, he joined the Department of Applied Physics 3, University of Seville, where he became an Associate Professor in 2004. His research interests include electromagnetic compatibility for power electronics and signal integrity and radiation effects in microwave integrated circuits and highspeed very large-scale integration (VLSI) interconnects.

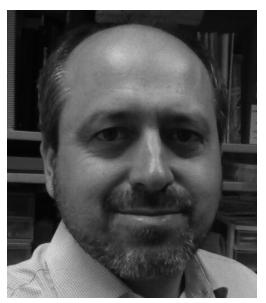

Manuel J. Freire (SM'14) received the Licenciado degree in Physics in 1995, and the Ph.D. degree in Physics in 2000, both from the University of Seville (Spain). He is Associate Professor in the Department of Electronics and Electromagnetism of the University of Seville. His research interests include leakage in microwave planar circuits, magnetostatic wave propagation in ferrite devices, frequency selective surfaces, subwavelength imaging with metamaterials, coils for magnetic resonance imaging, and EMC in power converters.

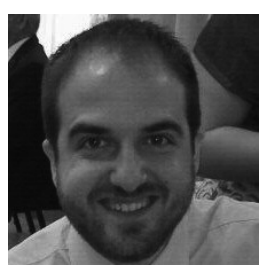

Sebastian Ramiro received the BS degree in industrial engineering and the MS degree in electronics and signal processing from the University of Seville, Spain, in 2009 and 2012 respectively. He joined the Electronics Technology Group of the University of Seville in 2010. From 2012 he is with Skylife Engineering as hardware and software engineer for embedded systems for airborne and test bench equipment. He is currently pursuing the Ph.D. degree in electromagnetic compatibility applied to aeronautical power converters. 